\title{
Hand and Body Blockage Measurements with Form-Factor User Equipment at $28 \mathrm{GHz}$
}

\author{
Vasanthan Raghavan, Sonsay Noimanivone, Sung Kil Rho, Bernie Farin, Patrick \\ Connor, Ricardo A. Motos, Yu-Chin Ou, Kobi Ravid, M. Ali Tassoudji, Ozge H. Koymen, and Junyi Li
}

\begin{abstract}
Blockage by the human hand/body is an important impairment in realizing practical millimeter wave wireless systems. Prior works on blockage modeling are either based on theoretical studies of double knife edge diffraction or its modifications, high-frequency simulations of electromagnetic effects, or measurements with experimental millimeter wave prototypes. While such studies are useful, they do not capture the form-factor constraints of user equipments (UEs). In this work, we study the impact of hand/body blockage with a UE at $28 \mathbf{G H z}$ built on Qualcomm's millimeter wave modem, antenna modules and beamforming solutions. We report five exhaustive and controlled studies with different types of hand holdings/grips, antenna types, and with directional/narrow beams. For both hard as well as loose hand grips, we report considerably lower blockage loss estimates than prior works. Critical in estimating the loss is the definition of a "region of interest" (RoI) around the UE where the impact of the hand/body is seen. Towards this goal, we define a RoI that includes the spatial area where significant energy is seen in either the no blockage or blockage modes. Our studies show that significant spatial area coverage improvement can be seen with loose hand grip due to hand reflections.
\end{abstract}

Index Terms-Millimeter wave, form-factor user equipment, measurements, hand blockage, body blockage, UE design, 5GNew Radio, 28 GHz.

\section{INTRODUCTION}

Over the last ten years, the interest in millimeter wave carrier frequencies has transformed from an academic/theoretical pursuit to commercial deployments. The first wave of commercial form-factor user equipments (UEs) are already available in the market with the physical layer operation conforming to the Third Generation Partnership Project (3GPP) standard specifications in Release 15. Despite this essentially mature background [1]-[11] in both the theory and practice of millimeter wave systems, there is still considerable and growing interest in understanding the performance limits of such systems imposed by the channel and propagation characteristics, radio frequency (RF) and hardware constraints and their impact and implications for low-cost, low-complexity and power-efficient physical layer design. The focus of this work is on one such impairment: blockage of millimeter wave signals at the UE end due to human hand and body.

Given that blockage is not a dominant impairment at sub-6 GHz carrier frequencies, a number of prior works have focussed on modeling blockage and understanding its implications on millimeter wave system performance. In particular, wireless standardization efforts at $60 \mathrm{GHz}$ for 802.11(ad) WiFi systems use ray-tracing studies to propose a human blockage model [12, Sections 3.3.8, 3.5.7, 5.3.9, 8]. This model reflects the probability that a blockage event happens, a distribution for blockage loss conditioned on it happening and time-scale modeling for blockage events. For cellular millimeter wave systems, the 3GPP TR38.901 [13. pp. 53-57] proposes a flat $30 \mathrm{~dB}$ loss over a defined blockage region for the UE in either the Portrait or Landscape modes. The loss region is modeled using data from studies with a form-factor experimental millimeter wave UE mockup/prototype at 28 and $60 \mathrm{GHz}$ and the loss is motivated by a survey of measurement studies with human/body blockage. The Mobile and wireless communications Enablers for the Twenty-twenty Information Society (METIS) project has proposed a human blockage model based on the double knife edge diffraction (DKED) framework in [14, pp. 39-41, 160162]. Human blockage measurements over a wideband setup at $60 \mathrm{GHz}$ has been considered in [15], where comparisons are made in terms of model fitting with the DKED and the uniform theory of diffraction (UTD) frameworks. Human blockage measurements using a $73 \mathrm{GHz}$ horn antenna setup is considered in [16]-[20] and substantial losses (30-40 dB) are reported.

In terms of form-factor studies, the impact of blockage at $15 \mathrm{GHz}$ is studied in [21] and subarray diversity is recommended for overcoming the deleterious effects of blockage. More recently, the loss with blockage is estimated in a prior work of ours [22] using a $28 \mathrm{GHz}$ form-factor prototype performing real-time beam switching/management and operating according to the system level specifications analogous to the 3GPP framework albeit with a proprietary subframe structure. This study reported an order-of-magnitude smaller blockage losses in beamformed systems than prior modeling efforts (e.g., $30 \mathrm{~dB}$ loss in TR38.901) and attributed the discrepancies in loss due to beamwidth differences between commercial-quality phased arrays and horn antennas (horn antennas have narrower beamwidths than UE-side phased arrays). In this study, blockage loss was estimated with overthe-air (OTA) measurements of beamformed received power differential between the no blockage and blockage scenarios. 
While such studies are useful in understanding the practical impact of blockage, the received power differential is a function of the channel environment (rich vs. non-rich multipath clusters) and the set of beam weights with which the link has been established between the base-station and the UE (which determines the dominant cluster in the channel excited in beamforming). Another important caveat common to all prior studies on blockage modeling is that they are either based on ray-tracing or electromagnetic simulation studies, or with experimental prototypes that may/may not be a form-factor implementation.

Contributions: In this context, this work reports blockage losses with a commercial form-factor UE operating at 28 $\mathrm{GHz}$. The UE is equipped with a commercial grade millimeter wave modem, antenna module solution and driven by a beam management software solution that adheres to the 3GPP system level protocol specifications in Rel. 15 [23]. The antenna module incorporated here uses a $4 \times 1$ dualpolarized patch array and two $2 \times 1$ dipole arrays across two polarizations/layers. Multiple commercial millimeter wave UEs available in the market today use similar antenna, modem and system level software implementations that realize low-overhead and low-complexity analog/RF beamforming and beam tracking [6], thereby improving signal range and coverage. Thus, this work is directly relevant in understanding blockage from a practical/implementation perspective.

In contrast to OTA measurements of [22], we report five controlled studies in an anechoic chamber that allows us to understand blockage by studying beam patterns over a sphere with Freespace/no blockage, a hand phantom used to emulate blockage, and a real/true human holding the phone with hand and body of the human blocking the signals. By studying the beam patterns over a sphere, the impact of the channel used to establish a beamformed link is removed and we can showcase the true impact of blockage in different directions. The reported studies correspond to different targeted antenna arrays of different dimensions $(4 \times 1$ patch array vs. $2 \times 1$ dipole array), different UE orientations (Portrait vs. Landscape), and different hand holdings/grips. The grips studied here include a "hard" hand holding grip where the hand completely engulfs all the antenna elements in the array with minimal air gaps between the fingers, a "loose" hand holding grip where only a few fingers engulf some of the antenna elements in the array with the remaining antenna elements seeing unobstructed signals, and an "intermediate" hand holding grip where a few fingers engulf some antenna elements with a big air gap between the palm of the hand and the remaining antenna elements.

From our studies, we note the following broad conclusions.

- We start with a gross estimate of blockage losses obtained by comparing the cumulative distribution functions (CDFs) of radiated signal power with Freespace/no blockage and with blockage. These gross loss estimates range from $8.5-17 \mathrm{~dB}$ in the hard hand grip mode to $3.5-11 \mathrm{~dB}$ in the loose hand grip mode for the $4 \times 1$ subarray. These loss estimates are significantly lower than loss estimates at 3GPP [13] and in prior studies [20]. The estimates provided here are also consistent with (and similar to) our prior work [22] that used a $28 \mathrm{GHz}$ experimental prototype and estimated a mean loss of $15.3 \mathrm{~dB}$ and $8.5 \mathrm{~dB}$ for hand and body blockage losses.

- Going further from all the prior works, we show that depending on the antenna type (dipole or patch), array size $(4 \times 1$ vs. $2 \times 1)$, type of beam used (scan angle and beamwidth), material property of UE, and the user's hand properties (such as hand grip, hand size, skin properties), etc.,

1) The hand can attenuate signals in a certain set of directions;

2) the hand can reflect energy in some set of directions; and

3) the hand can leave signal energy essentially unchanged in the remaining set of directions.

With a primary focus on blockage loss in prior works, the impact of hand reflections has not been explored. In this context, this work provides a first understanding of this aspect.

- The impact of hand/body in terms of signal deterioration varies with direction. Thus, to estimate the statistics of blockage loss, we need to define a "region of interest" (RoI) where blockage is observed. We begin with a RoI (denoted as $\mathcal{R}_{1}$ ) corresponding to the Freespace/no blockage region that is within a fixed signal threshold of its peak value. This RoI does not capture hand reflections into regions which had a poor signal strength in the no blockage mode. Thus, to consider the impact of hand reflections, we augment the above RoI (denoted as $\mathcal{R}_{5}$ ) with the region where signal strength in the blockage mode is also above a signal strength threshold. A number of other intermediate RoIs (denoted as $\mathcal{R}_{2}$, $\mathcal{R}_{3}$ and $\mathcal{R}_{4}$ ) are also defined corresponding to different nuanced aspects of blockage.

- We show that $\mathcal{R}_{1}$ is sufficient to capture the impact of blockage in scenarios with a hard hand grip where there are no prominent air gaps between fingers and hence there are minimal hand reflections. On the other hand, $\mathcal{R}_{5}$ is necessary to understand blockage in scenarios with a loose or intermediate hand grip, where a few antenna elements are unobstructed, or where a significant air gap can be seen between some fingers and the antenna elements leading to hand reflection gains.

- In general, studies with the hand phantom seem to be poorly correlated with true hand/body blockage performance. This may be attributed to the need for careful tuning of the hand phantom as well as its orientation and placement to correspond to how a real 
TABLE I

Summary Statistics From the Five Blockage Studies

\begin{tabular}{|c||c|c|c|c|c||c|}
\hline \hline Study & Subarray type & UE orientation & Hand grip & $\begin{array}{c}\text { Gross loss } \\
\text { estimate (in dB) }\end{array}$ & $\begin{array}{c}\text { Relative \% of } \\
\text { sphere lost }\end{array}$ & $\begin{array}{c}\text { Relative RoI } \\
\text { improvement (in \%) }\end{array}$ \\
\hline \hline 1 & $4 \times 1$ patch & Portrait & Hard & 8.6 to 17.2 & $66.7 \%$ to $85.3 \%$ & $2.1 \%$ to $4.6 \%$ \\
\hline 2 & $4 \times 1$ patch & Portrait & Loose & 3.6 to 10.6 & $36.0 \%$ to $43.9 \%$ & $6.7 \%$ to $8.0 \%$ \\
\hline 3 & $2 \times 1$ dipole & Portrait & Hard & 15.9 to 19.7 & $90.8 \%$ to $100.0 \%$ & $0 \%$ to $1.7 \%$ \\
\hline 4 & $2 \times 1$ dipole & Portrait & Loose & 0.4 to 10.8 & $20.0 \%$ to $25.7 \%$ & $8.5 \%$ to $12.9 \%$ \\
\hline 5 & $4 \times 1$ patch & Landscape & Intermediate & 9.5 to 12.7 & $68.6 \%$ to $99.9 \%$ & $8.9 \%$ to $15.7 \%$ \\
\hline \hline
\end{tabular}

hand holding works. Further, hand phantoms do not capture body effects. Differences in electromagnetic behavior induced by material property differences between the phantom material and the true human hand at millimeter wave carrier frequencies could also account for discrepancies in the hand phantom performance relative to the real hand. The use of hand phantoms requires more extensive studies in the future.

- The summary statistics in terms of blockage performance for these five studies are briefly described in Table II. These summary statistics illustrate the gross loss estimate (in $\mathrm{dB}$ ) with blockage across different spherical coverage levels, relative fraction of the sphere lost at different effective isotropically radiated power (EIRP) levels, and how hand reflections and the new definition of $\mathcal{R}_{5}$ relatively improves the RoI/spherical coverage (over $\mathcal{R}_{1}$ ).

This paper is organized as follows. Section III explains the experimental setup considered in this work in terms of hand/body blockage measurements. Section III considers the $4 \times 1$ patch subarray with a hard hand grip and studies the impact of hand/body blockage in careful detail by exploring the need for different RoIs in understanding the implications of blockage. Section IV performs similar studies for the four other scenarios considered in this work. Section $\mathrm{V}$ develops models for blockage for all the five scenarios considered here as well as compares the physical layer implications of this work with prior models on blockage. Section $\mathrm{VI}$ concludes the paper.

\section{EXPERIMENTAL SETUP}

We now explain the experimental setup used for measuring hand/body blockage in this paper.

\section{A. User Equipment}

The UE used in this study is equipped with a millimeter wave modem operating at $28 \mathrm{GHz}$ and using a 3GPP Release 15 spec-compliant software solution that performs intelligent beamforming and beam tracking. From an antenna module perspective, the UE consists of three modules denoted as Modules 1-3. These modules are equipped on the three edges/sides (two long edges and the top short edge) of the
UE. Each antenna module has a $4 \times 1$ dual-polarized patch array as well as two $2 \times 1$ dipole arrays that allows dualpolarized transmissions via two RF chains at $28 \mathrm{GHz}$. See Fig. 11a) for an illustration of the UE with the locations of the three antenna modules and the antenna setup within each module. Since the UE is a pre-commercial design, it has a width beyond $72 \mathrm{~mm}$ making it a wide-body phone design.

\section{B. Chamber Measurement Setup}

The anechoic chamber setup is pictorially illustrated in Fig. 11(b) and is now described. The measurement (receiving) antenna is an off-the-shelf dual-polarized broadband horn antenna (covering 18-40 GHz) with an antenna gain of $\approx$ $14 \mathrm{dBi}$ at $28 \mathrm{GHz}$. The $3 \mathrm{~dB}$ beamwidth in the $\mathrm{H}$ and $\mathrm{E}$ planes of the horn antenna at $28 \mathrm{GHz}$ are $35.8^{\circ}$ and $31.9^{\circ}$, respectively. The UE is placed on a fiberglass pedestal in the center of the chamber. The transmit power used with the active millimeter wave antenna module is $4 \mathrm{dBm}$, which is well within the 3GPP EIRP regulations for commercial millimeter wave devices and is intended for short distance coverage between the UE and the measurement antenna. The distance between the UE and the measurement antenna is $\approx 1.50$ meters ( 59 inches).

Short RF flex cables (with some loss) are used to connect the measurement antenna with a power meter. The power level observed by the power meter $\left(P_{\mathrm{rx}}\right)$ can be written as

$$
P_{\mathrm{rx}}=\mathrm{EIRP}_{\mathrm{tx}}+G_{\mathrm{rx}}-\text { Path loss }- \text { Loss }_{\mathrm{cable}},
$$

where EIRP $\mathrm{tx}_{\mathrm{x}}$ is the transmitted EIRP with reference plane set to the outer surface of the back cover ${ }^{1}$ of the UE side, $G_{\text {rx }}$ is the gain of the measurement antenna, and Path loss and Loss cable correspond to loss in OTA transmissions (with a path loss exponent of 2 since a line-of-sight path is maintained between the UE and the measurement antenna) and loss in the cables connecting the horn antenna with the power meter, respectively. To measure the received power level accurately, an OTA path loss calibration procedure is performed to capture the impact of measurement antenna gain, cable loss and path loss. Note that while EIRP $P_{t x}$

\footnotetext{
${ }^{1}$ The considered reference plane implies that the loss due to the radome/back cover is lumped with the measured data and thus, it is not necessary to worry about the angle-dependent radome loss.
} 


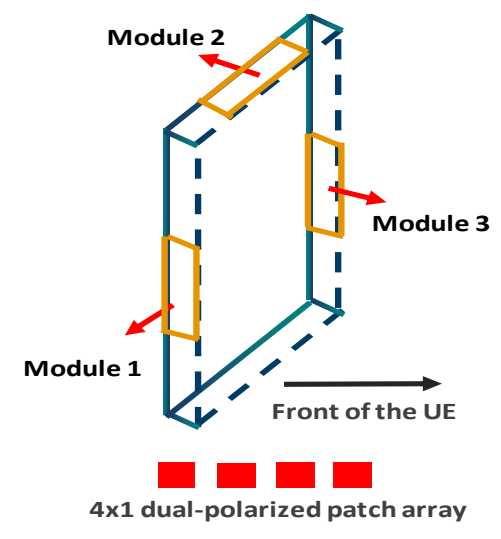

(a)

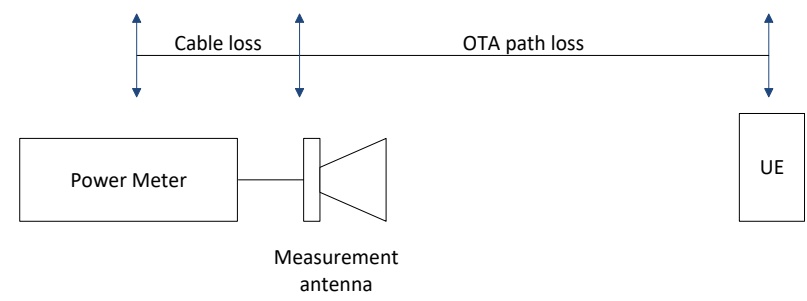

(b)

Fig. 1. (a) Pictorial illustration of the relative positions of the three antenna modules in the UE. (b) Pictorial illustration of the measurement setup.

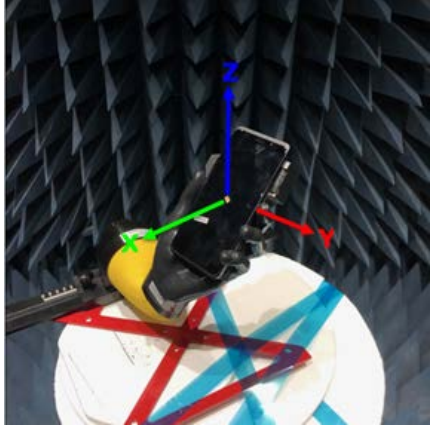

(a)

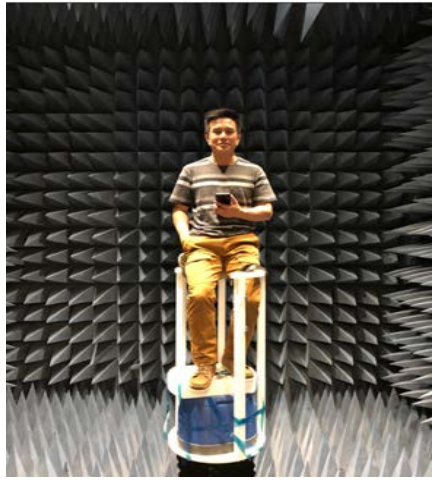

(d)

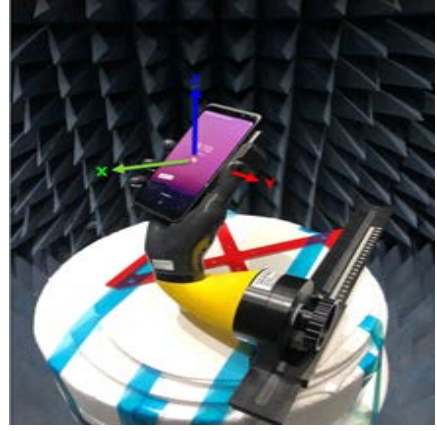

(b)



(e)

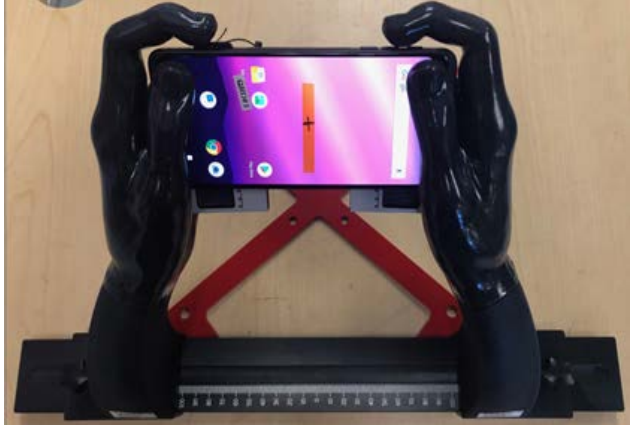

(c)



(f)

Fig. 2. Illustration of hand phantom and a true hand placed on the left hand side of the UE ((a) and (d)) and right hand side of the UE ((b) and (e)), both in Portrait mode. (c) and (f): Hand phantom and true hand in gaming/Landscape mode.

can be estimated theoretically, measurements are needed to understand the over-/under-estimation of single antenna elemental gains and the array gains due to different sets of beam weights in different directions, as well as non-idealities in the array geometry and impact of UE material properties on the observed beam patterns.
As the UE transmits with a certain set of beam weights, the measurement antenna is rotated (control for the rotation is driven by an automated software) at $\approx 5^{\circ}$ steps in azimuth and elevation. Due to the design of the chamber, a limited $170^{\circ}$ (of the possible $180^{\circ}$ ) coverage in elevation is possible leading to a coverage map of the beam pattern over the 
$360^{\circ} \times 170^{\circ}$ part of the sphere. By carefully choosing the UE orientation in Freespace and with blockage, the impact of the missing $10^{\circ}$ in elevation plane on the conclusions of this work can be made minimal. In the tests conducted in this paper, the UE orientation in the testing positions means that the missing $10^{\circ}$ is towards the bottom of the UE which has no antenna module coverage and is covered by side lobes of other antenna modules. Since the EIRP in these side lobe directions is expected to be low, lack of measurements in the missing $10^{\circ}$ is expected to affect the tail of the performance curves, which do not carry any major impact on understanding the implications of blockage.

\section{Hand Phantom and True Hand Holding}

In the Portrait mode, we first use an anthropomorphic hand phantom which is specifically designed for evaluating and optimizing OTA performance of ultra-wide mobile phone devices (defined as having a width between 72 and 92 $\mathrm{mm}$ ). The hand phantom is manufactured using a siliconecarbon-based mixture with material properties conforming to the Cellular Telecommunications Industry Association (CTIA) definitions and standards for hand phantoms. The use of a special low-loss silicone coating extends its useable frequency range from $3 \mathrm{GHz}$ to $110 \mathrm{GHz}$. A low-loss highprecision data mode fixture is used for accurate and stable positioning of the hand phantoms in the correct position for the testing of devices. In the Landscape mode, we use a two-handed grip for gaming mode studies in conjunction with a wrist extension and the data mode fixture. The hand phantoms on the left and right edges of the UE in Portrait mode and the hand phantom in the gaming/Landscape mode are illustrated in Figs. 22(a)-(c), respectively.

For the true hand holding tests, a testing person holds the UE and sits in a static position in the chamber while the measurements are conducted. Each test (scan over the sphere) takes approximately 18-21 minutes and thus different testing persons are employed in the studies in this paper. The testing persons vary from having a small body size (145 lbs, 5 feet and 4 inches and $165 \mathrm{lbs}, 5$ feet and 1 inch) to a large body size (214 lbs, 6 feet and 1 inch). Our studies show that while the blockage losses reported in this paper have some minor dependencies on the size of the hand, palm and fingers as well as the skin properties of the hand, these dependencies are secondary and minor in comparison with the type of hand holding/grip relative to the antenna array dimensions involved and the steering direction of the beams used.

In terms of hand holding, three broad categories of tests are identified:

- A hard hand grip with the right hand in the Portrait mode that completely engulfs all the antenna elements in Module 3 with minimal air gaps between the fingers.

- A loose hand grip with the left hand in the Portrait mode where only a few fingers engulf some of the antenna elements in Module 3 with unobstructed signals from other antenna elements.

- An intermediate hand grip with two hands in the Landscape mode where a few fingers engulf some antenna elements in Module 2 with a big air gap between the palm of the hand and the remaining antenna elements.

These three hand grips are reflective of the testing person's holding of the UE as illustrated in Figs. 2(d)-(f).

\section{Study 1: $4 \times 1$ PATCH SUbARRAY With A HARD HAND GRIP}

The deployment of multiple antennas at millimeter wave carrier frequencies can be leveraged to improve the link margin via beamforming. Since a limited number of RF chains are available at the UE end at millimeter wave carrier frequencies (the UE considered in this work has two RF chains, which are used for polarization-based transmissions), increased array gain is realized with analog/RF beamforming. Here, a three-bit phase shifter and a variable gain amplitude control are used at each antenna element to cophase the signals along desired/pre-specified directions. A beamforming scheme realized with a finite-sized analog/RF beam codebook of beam weights that steers energy along the dominant cluster(s) in the channel is a good low-complexity near-optimal solution relative to the optimal beamforming scheme (performing maximum ratio combining) [6]. The performance of this codebook improves as the codebook size increases and approaches the performance of a directional beamforming scheme with perfect knowledge of the dominant cluster in the channel as seen at the base-station and UE ends [24]. In this section, we study the beamforming performance of the analog codebook of beams (without/with blockage) for the $4 \times 1$ patch subarray in Module 3 with a hard hand grip. Other subarrays and hand holdings are considered in Sec. IV

\section{A. Beamforming Performance}

To understand the implications of blockage, we consider a codebook of three beams for the $4 \times 1$ subarray. We consider a small codebook size of three since practical UE codebook constraints are determined by low latency requirements for initial link acquisition [6]. In the case of the $4 \times 1$ patch subarray, these three beams are chosen to steer energy along the boresight of the array, $+30^{\circ}$ to the boresight and $-30^{\circ}$ to the boresight, respectively. The beam patterns in Freespace (over a sphere around the UE) are illustrated for these three beams in Figs. 3 a)-(c), respectively. Note that in addition to the correct orientation of the beam patterns (relative to the coordinate system in Fig. 2(b)), the beam pattern of each beam is reasonably regular and conforming to its theoretically expected performance [25]. Further, the beamwidth of each beam is $\approx 25^{\circ}-30^{\circ}$ suggesting that these three beams can cover a $75^{\circ}-90^{\circ}$ spatial area in one 


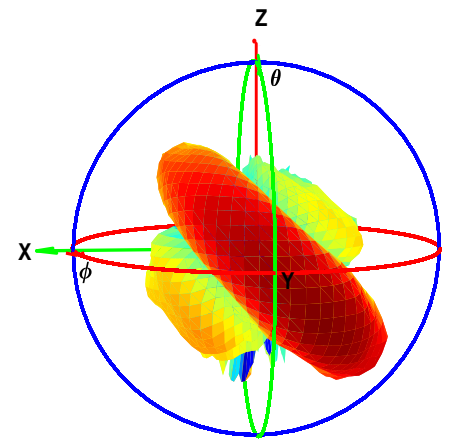

Max EIRP ${ }_{\mathrm{Rx}}=-\mathbf{2 5 . 8 \mathrm { dBm }}$

(a)

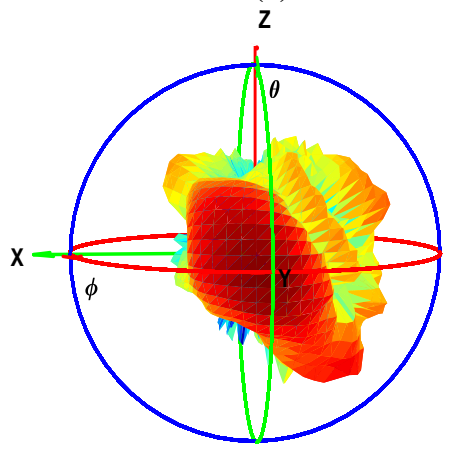

Max EIRP ${ }_{\mathrm{Rx}}=-25.3 \mathrm{dBm}$

(d)

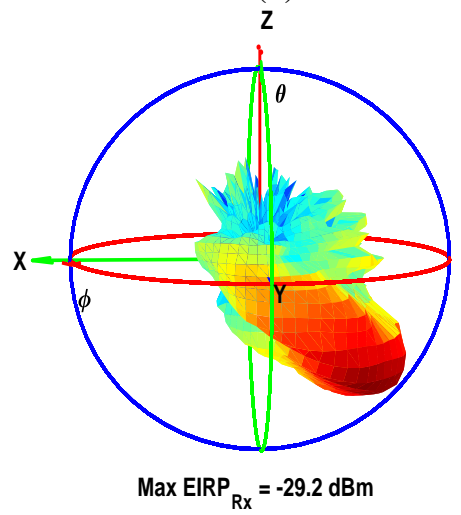

(g)
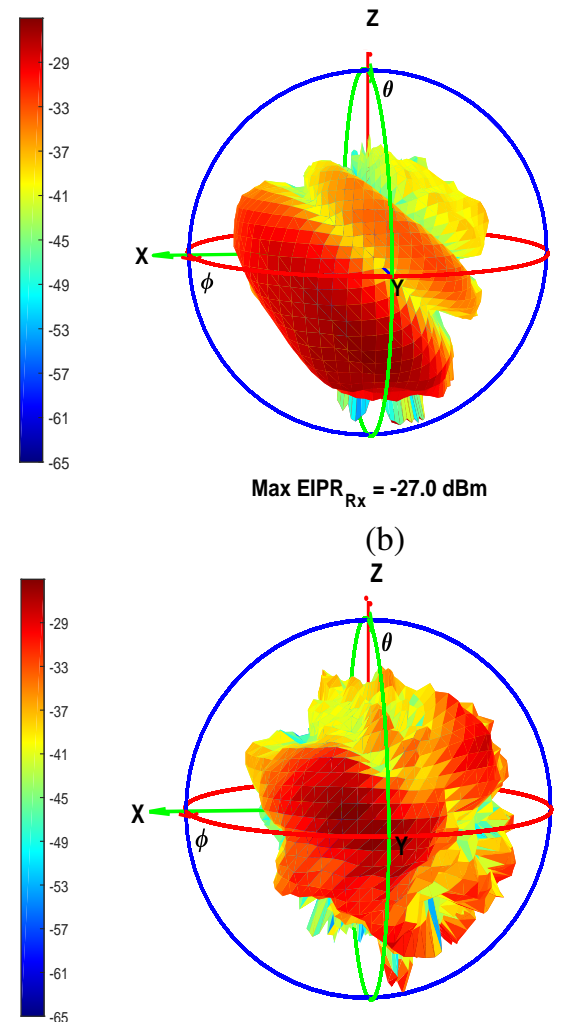

Max EIRP ${ }_{\mathrm{Rx}}=-29.3 \mathrm{dBm}$

(e)

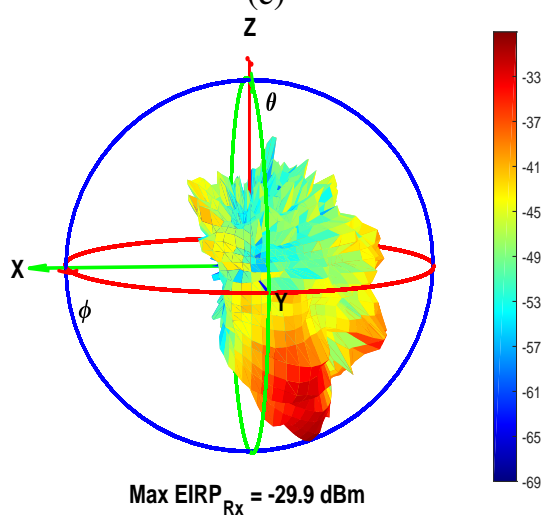

(h)



$\operatorname{Max}_{\text {EIRP }_{\mathrm{Rx}}}=-25.7 \mathrm{dBm}$

(c)



Max EIRP ${ }_{\mathrm{Rx}}=-24.6 \mathrm{dBm}$

(f)

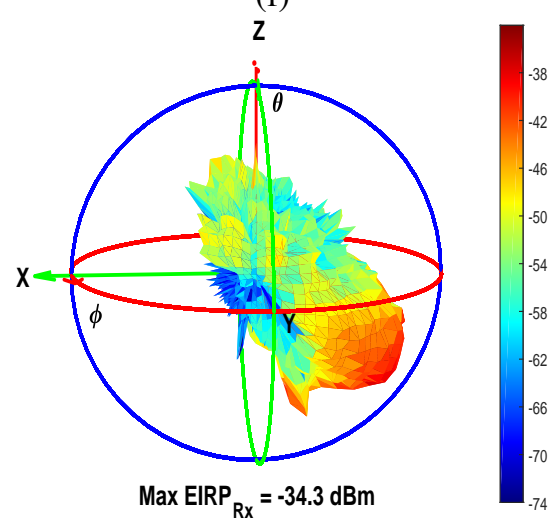

(i)

Fig. 3. Beam patterns in (a)-(c) Freespace, (d)-(f) with a hand phantom and (g)-(i) with a true hand holding for the three beams considered for the $4 \times 1$ patch subarray.

TABLE II

EIRP-BASED COMPARISONS FOR THE HARD HAND GRIP (STUDY 1)

\begin{tabular}{|c|c|c|c|c|c|c|c|c|c|c|c|}
\hline \multirow{6}{*}{$\begin{array}{l}\vec{z} \\
\text { D্ } \\
\text { in }\end{array}$} & \multirow{3}{*}{$\begin{array}{c}\text { EIRP } \\
\text { (in } \mathrm{dBm} \text { ) }\end{array}$} & \multicolumn{7}{|c|}{ Spherical Coverage Lost } & \multicolumn{3}{|c|}{ Loss (in $\mathrm{dB}$ ) } \\
\hline & & \multirow{2}{*}{$\begin{array}{c}\text { Freespace } \\
\text { (in \%) }\end{array}$} & \multicolumn{3}{|c|}{ Hand Phantom (in \%) } & \multicolumn{3}{|c|}{ True Hand (in \%) } & \multirow{2}{*}{$\begin{array}{c}\text { Percentile } \\
90\end{array}$} & \multirow{2}{*}{$\begin{array}{c}\text { Hand Phantom } \\
3.1\end{array}$} & \multirow{2}{*}{$\begin{array}{c}\text { True Hand } \\
8.6\end{array}$} \\
\hline & & & & Abs. & Rel. & & Abs. & Rel. & & & \\
\hline & $>-35$ & 23.3 & 13.5 & 9.7 & 41.8 & 3.4 & 19.8 & 85.3 & 80 & 3.4 & 10.6 \\
\hline & $>-40$ & 41.8 & 28.9 & 12.9 & 31.0 & 12.1 & 29.7 & 71.0 & 50 & 3.4 & 12.2 \\
\hline & $>-45$ & 65.0 & 48.5 & 16.6 & 25.5 & 21.7 & 43.4 & 66.7 & 20 & 2.0 & 17.2 \\
\hline
\end{tabular}


dimension, which is typically the coverage area of a linear array at millimeter wave carrier frequencies.

In a beamformed realization, an overlay plot of the beam pattern over the sphere due to the best of the three beams is important. Such a characterization, by way of comparison without and with blockage, also allows us to understand the impact of blockage on beamformed performance. Fig. 4(a) illustrates this overlay plot for the $4 \times 1$ patch subarray in Freespace. In this plot, the behavior over the sphere is plotted as a two-dimensional plane (over $\phi-\theta$ where $\phi$ and $\theta$ are the azimuth and elevation angles, respectively). From this plot, we observe that this subarray is well-designed to ensure good coverage over at least a $90^{\circ} \times 60^{\circ}$ coverage region in Freespace, which is typical for antennas at millimeter wave carrier frequencies.

We next consider the behavior of the beam weights used with the patch subarray with the hand phantom and a true hand holding the UE. Figs. 3(d)-(f) and (g)-(i) plot the beam patterns of the three beams in these two setups, respectively. The overlay plots of the best of the three beams' beam patterns are plotted in these two scenarios in Figs. 4(b) and (c), respectively. Note that Fig. 4(b) shows that some beams have better performance with the hand phantom than in Freespace which is possible due to reflections by the hand phantom, whereas Fig. 4(c) shows that the impact of the beam which is at $-30^{\circ}$ off boresight is rarely observed.

From these plots, we also observe that while the hand phantom irregularly distorts the beam patterns in Freespace, this distortion is still rather minor. In particular, the peak EIRP is distorted by $0.5 \mathrm{~dB}, 2.3 \mathrm{~dB}$ and $1.1 \mathrm{~dB}$ (gains in some cases) for the three beams, respectively. On the other hand, the distortion with a true hand holding is significant (peak EIRP distortion of $3.4 \mathrm{~dB}, 2.9 \mathrm{~dB}$ and $8.6 \mathrm{~dB}$ ) with considerable signal deterioration observed over the beam patterns in Freespace. Specifically, the beam which is $+30^{\circ}$ off boresight steers energy towards the torso, hips and stomach of the human holding the UE and is thus less impacted in terms of signal distortion relative to the beam which is $-30^{\circ}$ off boresight, which steers energy towards the face and shoulder and is thus significantly impacted by the human. The wide variations between the hand phantom behavior and a true human holding the UE indicates that the use of the hand phantom to replicat ${ }^{2}$ true hand holding studies is risky in terms of drawing meaningful lessons. This is because the hand phantom can at best replicate the effects of the hand with careful tuning, but not capture the effects

\footnotetext{
${ }^{2}$ Blockage studies with non-human intervention in the lab/chamber at millimeter wave frequencies would be of great interest if the hand phantom results faithfully replicate true hand holding results. The studies in this paper show that there is a considerable gap between these two sets of studies, which justifies the need for further work in careful tuning of the hand phantom to match the results from true hand holding studies. At the very least, more studies are necessary to understand if/when hand phantoms can replicate true hand holding results.
}

of the body. Thus, true hand/body-based blockage studies are necessary to understand the implications of blockage.

\section{B. Estimating the Loss Region}

To understand the implications of beamforming, we now plot the CDFs of the EIRP as seen over the sphere (weighted by $\sin (\theta)$ ) in Freespace and with the hand (both hand phantom and the true hand holding). The weighting by $\sin (\theta)$ is essential since the sample points in the $\phi-\theta$ plane are uniform (at steps of $5^{\circ}$ ) leading to a crowding of points near the poles, which needs to be adjusted by the Jacobian of the coordinate transformation from a Cartesian/rectangular coordinate system to a spherical coordinate system [26]. This plot in Fig. 4(d) shows that there are two ways to interpret the $\mathrm{CDF}$ data. In the first view, the true hand holding leads to a 20\%-45\% (absolute) spherical coverage loss at different EIRP levels. In the second view, the true hand leads to a signal strength degradation of $8.5-17 \mathrm{~dB}$ at different percentile points. Reinforcing the prior observations on the mismatch of the hand phantom in capturing blockage performance, we note that the hand phantom leads to a $9.5 \%$ $17 \%$ (absolute) spherical coverage loss at different EIRP levels, or an equivalent 2-3.5 dB loss at different percentile points. Table III provides a summary of the absolute and relative spherical coverage losses at different EIRP levels as well as the loss seen at different percentile points.

Given such a wide range of losses at different percentile points, it is reasonable to ask as to what is a good model for spherical coverage loss and/or blockage loss. We now deal with this question in more detail. To understand the impact of blockage, two broad questions are laid out in prior works:

- What is the Region-of-Interest (RoI) in terms of blockage's impact?

- What is the loss seen over this RoI? Can this loss be modeled as an appropriate stochastic distribution?

In the sequel, we show that the above view is quite simplistic in terms of characterizing blockage performance. Specifically, we show that blockage does not just lead to losses over the RoI, but can also lead to gains due to reflection of signals from the fingers, palm and the hand. The precise nature and scale of the reflection gains depends on the type of hand grip and orientation, user-specific skin properties, etc. Thus, to truly understand the implications of blockage in terms of physical layer performance, we need to define the RoI carefully. Towards this goal, we define multiple such RoIs and show the broad utility of two specific RoI definitions.

Let $G(\theta, \phi)$ denote the beamforming array gain (in $\mathrm{dB}$ ) seen in Freespace in a certain direction $(\theta, \phi)$. Let $G_{\max }$ (also in $\mathrm{dB}$ ) denote the maximum gain in Freespace over all directions. That is,

$$
G_{\max }=\max _{\theta, \phi} G(\theta, \phi)
$$






(a)

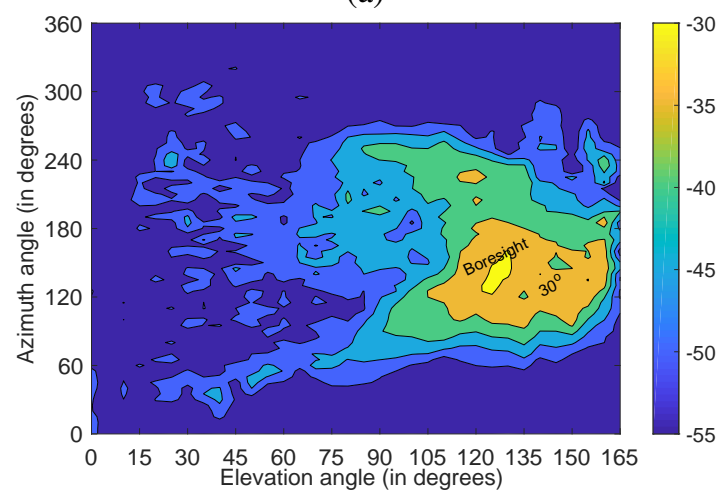

(c)



(b)

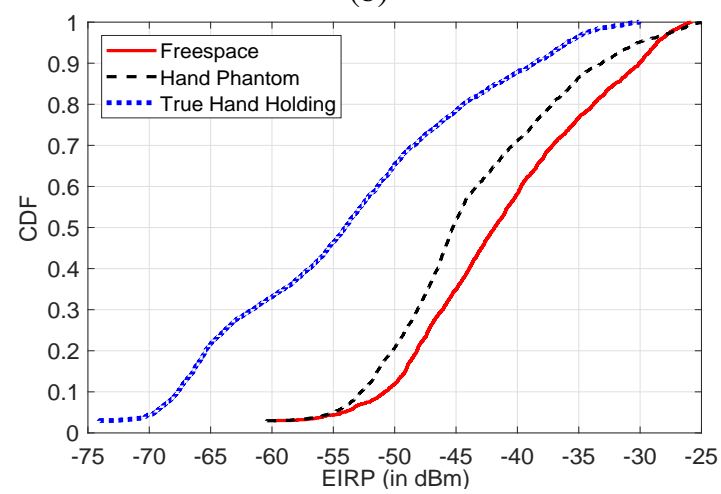

(d)

Fig. 4. (a)-(c) Overlay plots of the best of the three beam patterns in Freespace, with hand phantom and true hand holding for the $4 \times 1$ patch subarray. (d) CDFs of EIRP for different modes in the $4 \times 1$ patch subarray.

The typical definition of a RoI for a certain choice of threshold $\Delta_{1}$ (in $\mathrm{dB}$ ) is:

$$
\mathcal{R}_{1}\left(\Delta_{1}\right)=\left\{(\theta, \phi): G(\theta, \phi) \geq G_{\max }-\Delta_{1}\right\} .
$$

That is, $\mathcal{R}_{1}\left(\Delta_{1}\right)$ captures the region where the Freespace gains are within a fixed cutoff $\left(\Delta_{1} \mathrm{~dB}\right)$ of $G_{\max }$. It is important to note that this definition of $\mathcal{R}_{1}$ only relies on the Freespace gain, and not on what happens with hand/body blockage (which is quite naïve from understanding the implications of blockage). Further, this region does not have to be a rectangular/regular region in $(\theta, \phi)$ nor does it have to be a single connected region. In general, $\mathcal{R}_{1}\left(\Delta_{1}\right)$ could be a union of multiple irregular regions.

We now consider multiple ways in which blockage performance can be incorporated into the definition of a RoI by enhancing $\mathcal{R}_{1}\left(\Delta_{1}\right)$. Let $G_{\text {body }}(\theta, \phi)$ and $G_{\text {max,body }}$ denote the gain seen with hand/body holding the UE in a direction $(\theta, \phi)$ and the maximum of this gain over all $(\theta, \phi)$. We can define the following four RoIs for different choices of
$\Delta_{2}, \Delta_{3}, \Delta_{4}$ and $\Delta_{5}:$

$$
\begin{aligned}
\mathcal{R}_{2}\left(\Delta_{1}, \Delta_{2}\right)= & \left\{(\theta, \phi): G(\theta, \phi) \geq G_{\max }-\Delta_{1}\right. \text { or } \\
& \left.G_{\text {body }}(\theta, \phi) \geq G_{\max , \text { body }}-\Delta_{2}\right\} \\
\mathcal{R}_{3}\left(\Delta_{1}, \Delta_{3}\right)= & \left\{(\theta, \phi): G(\theta, \phi) \geq G_{\max }-\Delta_{1}\right. \text { or } \\
& \left.G_{\text {body }}(\theta, \phi) \geq G_{\max }-\Delta_{3}\right\} \\
\mathcal{R}_{4}\left(\Delta_{1}, \Delta_{4}\right)= & \left\{(\theta, \phi): G(\theta, \phi) \geq G_{\max }-\Delta_{1}\right. \text { or } \\
& \left.G_{\text {body }}(\theta, \phi) \geq \Delta_{4}\right\} \\
\mathcal{R}_{5}\left(\Delta_{5}\right)= & \left\{(\theta, \phi): G(\theta, \phi) \text { or } G_{\text {body }}(\theta, \phi) \geq \Delta_{5}\right\} .
\end{aligned}
$$

The intuitive meaning of these RoIs is that in addition to the region captured by $\mathcal{R}_{1}$, they capture the following:

- $\mathcal{R}_{2}$ captures the additional region where the body reflects power.

- $\mathcal{R}_{3}$ captures the additional region where the body reflects power as long as that power is viable from a Freespace perspective.

- $\mathcal{R}_{4}$ captures the additional region where the body reflects power that is nominally good as described by $\Delta_{4}$ (where this $\Delta_{4}$ parameter is typically chosen to meet some link budget constraint).

- $\mathcal{R}_{5}$ captures the region where either Freespace- or hand/body blockage-based signals are good as de- 


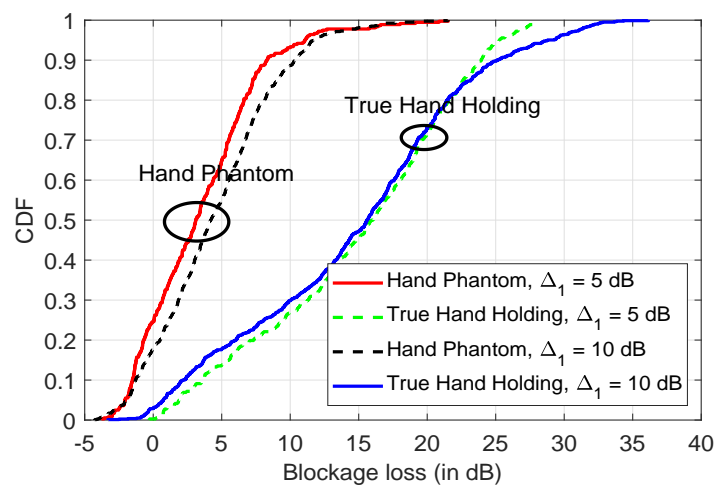

(a)



(c)

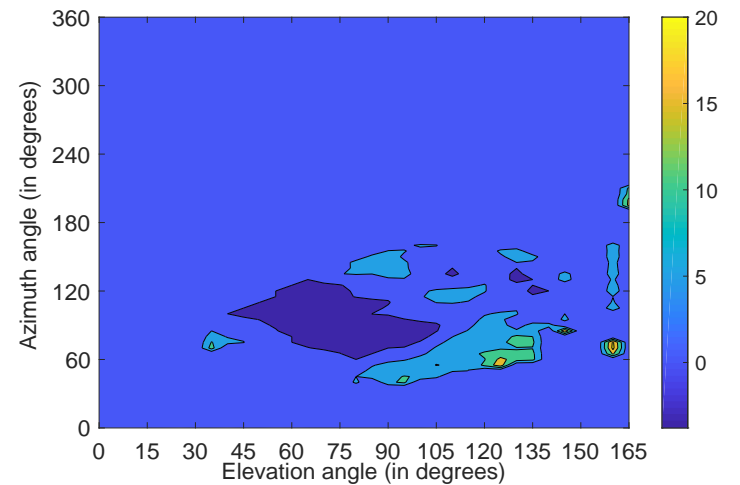

(b)

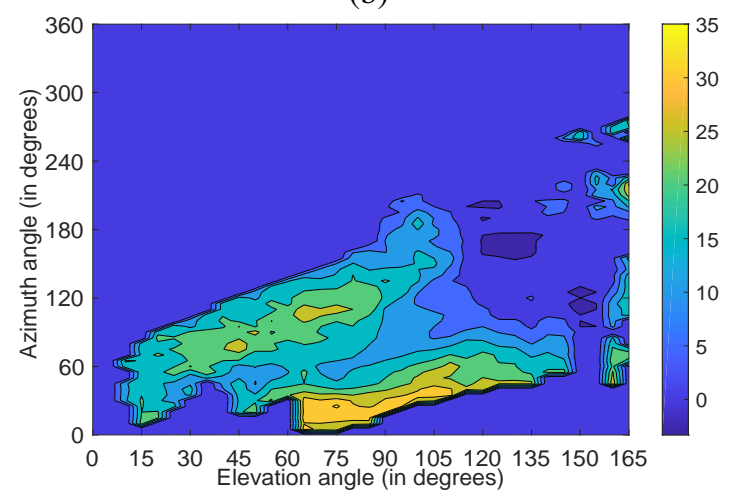

(d)

Fig. 5. (a) CDFs of blockage loss with the hard hand grip over $\mathcal{R}_{1}$ with $\Delta_{1}=5$ and $10 \mathrm{~dB}$. Blockage behavior over the sphere with (b) hand phantom and (c) true hand holding for $\mathcal{R}_{1}$ with $\Delta_{1}=5 \mathrm{~dB}$. (d) Blockage behavior over the sphere with true hand holding for $\mathcal{R}_{1}$ with $\Delta_{1}=10 \mathrm{~dB}$.

scribed by the common link margin threshold $\Delta_{5}$ (which is chosen to meet some link budget constraint).

Note that for $\Delta_{2}=\Delta_{3}, \mathcal{R}_{3}$ corresponds to a more aggressive definition of RoI relative to $\mathcal{R}_{2}$ since $G_{\max } \geq G_{\text {body,max }}$. On the other hand, the definitions of $\mathcal{R}_{4}$ and $\mathcal{R}_{5}$ strike a balance between the definitions of $\mathcal{R}_{2}$ and $\mathcal{R}_{3}$ with appropriate choices of $\Delta_{4}$ and $\Delta_{5}$. Also, note that $\mathcal{R}_{4}$ and $\mathcal{R}_{5}$ rely on link budget-driven parameters, while $\mathcal{R}_{2}$ and $\mathcal{R}_{3}$ do not. That said, by appropriately tuning $\Delta_{2}, \Delta_{3}$ and $\Delta_{4}$ (in a non-link budget driven basis), $\mathcal{R}_{2}, \mathcal{R}_{3}$ and $\mathcal{R}_{4}$ can be made comparable with each other.

We now present the CDFs of the EIRP differential between the Freespace and true hand holding scenarios for the RoI corresponding to $\mathcal{R}_{1}$ with $\Delta_{1}=5 \mathrm{~dB}$ and $\Delta_{1}=10 \mathrm{~dB}$ in Fig. 5(a). The RoI $\mathcal{R}_{1}$ with $\Delta_{1}=5 \mathrm{~dB}$ captures $\approx 14.6 \%$ of the sphere with a mean, median and standard deviation of the loss with true hand holding being $14.9 \mathrm{~dB}, 16.0 \mathrm{~dB}$ and $7.3 \mathrm{~dB}$, respectively. Similarly, for $\mathcal{R}_{1}$ with $\Delta_{1}=10$ $\mathrm{dB}$, the RoI captures $\approx 32.6 \%$ of the sphere with the mean, median and standard deviation of the loss with true hand holding being $14.7 \mathrm{~dB}, 15.6 \mathrm{~dB}$ and $8.4 \mathrm{~dB}$, respectively. In both scenarios, there is a wide discrepancy in terms of the CDF behavior for the true hand holding relative to the hand phantom (which is not entirely surprising). Further, the EIRP differential over these regions for the best of the three beams are plotted in the $\phi-\theta$ plane in Figs. 5(b)-(d), respectively. These plots correspond to the hand phantom's behavior with $\Delta_{1}=5 \mathrm{~dB}$ and true hand holding for $\Delta_{1}=5$ and $10 \mathrm{~dB}$, respectively. Clearly, these plots show that the true hand holding behavior seen over $\mathcal{R}_{1}$ is mostly loss, which is typical of blockage performance characterization in prior works.

For other RoIs, Fig. 6 provides a representative plot of blockage performance with some choices of parameters defining these RoIs. From these plots, we observe that there are some regions of the sphere where hand reflections can lead to substantial gains (regions marked in ellipses in Fig. 6). Based on the new RoI definitions, the mean, median, standard deviation of blockage loss and the RoI's coverage area in the sphere are described in Table III From this table, we note that $\mathcal{R}_{2}$ and $\mathcal{R}_{4}$ describe more reflection gains with reduced values of blockage loss (compared with $\mathcal{R}_{1}$ ). On the other hand, $\mathcal{R}_{3}$ and $\mathcal{R}_{5}$ are comparable with $\mathcal{R}_{1}$. These behaviors are specific to the parameters used for understanding blockage behavior (note that $\mathcal{R}_{2}, \mathcal{R}_{3}$ and $\mathcal{R}_{4}$ can be made comparable by careful parameter tuning).

Note that $\mathcal{R}_{1}$ corresponds to the RoI with just Freespace information alone, whereas $\mathcal{R}_{5}$ corresponds to Freespace as 


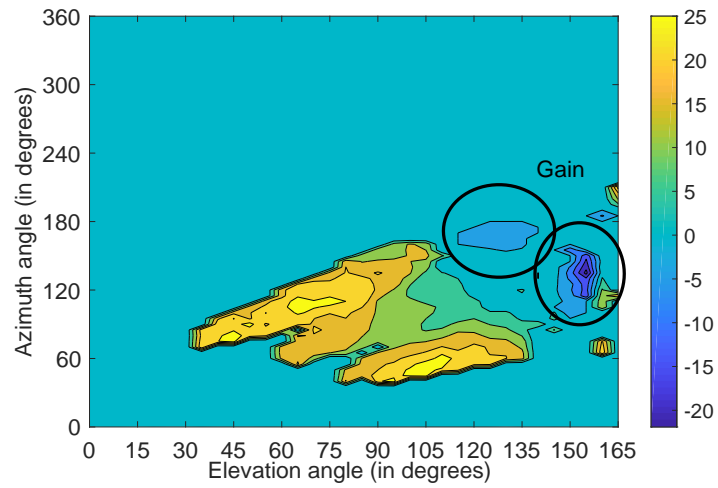

(a)

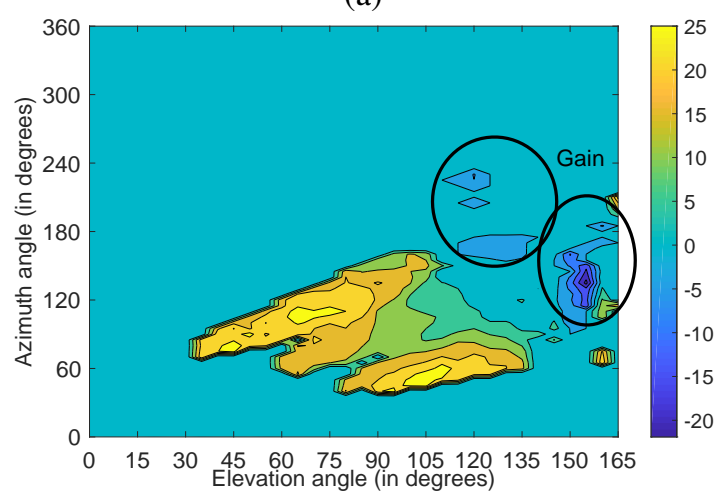

(c)

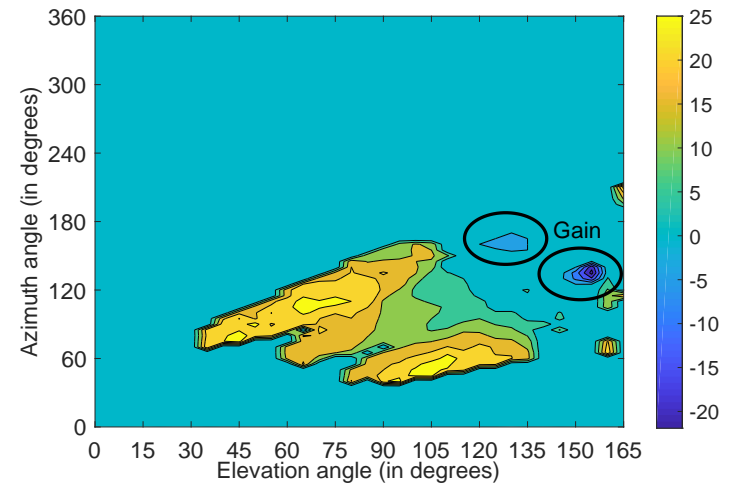

(b)

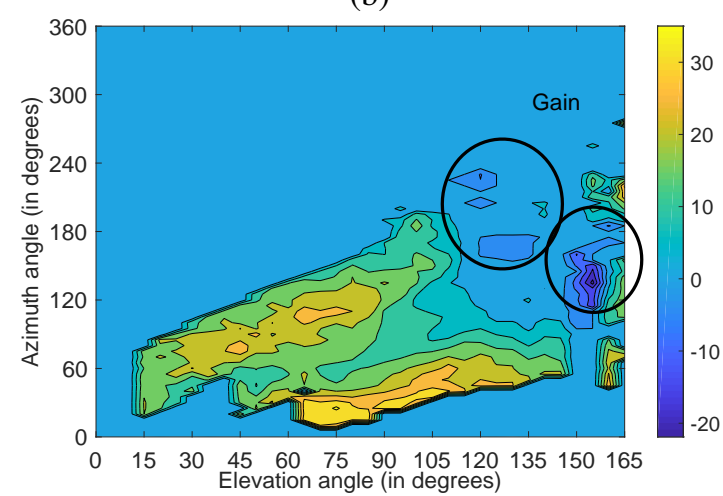

(d)

Fig. 6. CDFs of blockage loss with the hard hand grip over (a) $\mathcal{R}_{2}$ with $\Delta_{1}=\Delta_{2}=5 \mathrm{~dB}$, (b) $\mathcal{R}_{3}$ with $\Delta_{1}=\Delta_{3}=5 \mathrm{~dB}$, (c) $\mathcal{R}_{4}$ with $\Delta_{1}=5$ $\mathrm{dB}$ and $\Delta_{4}=-35 \mathrm{dBm}$, and (d) $\mathcal{R}_{5}$ with $\Delta_{5}=-35 \mathrm{dBm}$.

well as hand/body information. In general, we are interested in performing a head-to-head comparison between these two RoIs as they generally capture how RoI is defined in prior works and how RoI can be modified by incorporating blockage information. For this head-to-head comparison, we choose $\Delta_{1}$ and $\Delta_{5}$ to ensure that the EIRP is below a fixed level in both cases (in this comparison, we choose $-35,-40$ and $-45 \mathrm{dBm}$ as benchmarks). Table III compares $\mathcal{R}_{1}$ with $\mathcal{R}_{5}$ and shows that $\mathcal{R}_{5}$ only improves the area of interest on an absolute scale by $1 \%-2.5 \%$ (relatively from $2 \%-4.5 \%$ ) of the sphere. This is not a substantial increase in coverage area and the reason for this small increase is that there are hardly any reflection gains in the hard hand grip mode over any part of the sphere. Thus, in this case, $\mathcal{R}_{1}$ is sufficient to capture blockage performance. However, we will see in Sec. IV] that $\mathcal{R}_{5}$ and other RoIs become useful with looser hand grips.

\section{Beamforming Performance of Other SUBARRAYS AND HAND HOLDINGS}

We now present results from Studies 2-5 which cover other subarrays and hand holdings.

\section{A. Study 2: $4 \times 1$ Patch Subarray with a Loose Hand Grip}

We start with the same $4 \times 1$ patch subarray in Module 3 (as in Study 1), but with a left hand holding which ensures that only a few fingers cover the antennas in the module. As a result, we see blockage performance with an essentially loose hand grip mode.

Fig. 7 (a) illustrates the overlay plot of the best of the three beams used with this subarray under the loose hand grip mode. The close similarity between this plot and the Freespace plot in Fig. 4(a) suggests that the impact of the loose hand grip on blockage loss is significantly smaller than in the hard hand grip mode. Reflecting this observation, the CDF comparison of EIRPs in Fig. 7(b) and Table IV show that $10 \%-25 \%$ of the spherical coverage is lost at different EIRP levels, or an equivalent 3.5-10.5 dB loss at different spherical coverage levels. On the other hand, the hand phantom shows only a $5 \%-8 \%$ loss of spherical coverage, which is grossly mismatched to the observations with the true hand. The corresponding numbers for the hard hand grip are $20 \%-45 \%$ and $8.5-17 \mathrm{~dB}$, respectively, showing that the hand grip has a significant impact on the blockage loss observed. To understand the efficacy of an enhanced blockage region such as $\mathcal{R}_{5}$, we perform the same head-tohead comparison between these two RoIs as in the hard hand 
TABLE III

Blockage Performance With DifFerent RoIs For the Hard HAND GRIP (STUdy 1)

\begin{tabular}{|c||c|c|c|c||}
\hline Criterion & Mean (in dB) & Median (in dB) & Std. deviation (in dB) & Percentage of sphere \\
\hline $\mathcal{R}_{1}, \Delta_{1}=5 \mathrm{~dB}$ & 14.9 & 16.0 & 7.3 & 14.6 \\
\hline $\mathcal{R}_{1}, \Delta_{1}=10 \mathrm{~dB}$ & 14.7 & 15.6 & 8.4 & 32.6 \\
\hline $\mathcal{R}_{2}, \Delta_{1}=\Delta_{2}=5 \mathrm{~dB}$ & 12.6 & 14.3 & 9.3 & 16.7 \\
\hline $\mathcal{R}_{2}, \Delta_{1}=5 \mathrm{~dB}, \Delta_{2}=10 \mathrm{~dB}$ & 8.9 & 8.3 & 10.3 & 22.7 \\
\hline $\mathcal{R}_{3}, \Delta_{1}=\Delta_{3}=5 \mathrm{~dB}$ & 14.4 & 15.7 & 8.1 & 14.9 \\
\hline $\mathcal{R}_{3}, \Delta_{1}=5 \mathrm{~dB}, \Delta_{3}=10 \mathrm{~dB}$ & 11.0 & 12.6 & 9.9 & 19.0 \\
\hline $\mathcal{R}_{4}, \Delta_{1}=5 \mathrm{~dB}, \Delta_{4}=-35 \mathrm{dBm}$ & 11.9 & 13.7 & 9.6 & 17.7 \\
\hline $\mathcal{R}_{4}, \Delta_{1}=5 \mathrm{~dB}, \Delta_{4}=-40 \mathrm{dBm}$ & 8.8 & 7.6 & 9.3 & 23.3 \\
\hline $\mathcal{R}_{4}, \Delta_{1}=5 \mathrm{~dB}, \Delta_{4}=-45 \mathrm{dBm}$ & 7.6 & 6.1 & 9.5 & 31.2 \\
\hline $\mathcal{R}_{5}, \Delta_{5}=-35 \mathrm{dBm}$ & 13.9 & 15.4 & 9.9 & 30.8 \\
\hline $\mathcal{R}_{5}, \Delta_{5}=-40 \mathrm{dBm}$ & 14.2 & 15.4 & 9.6 & 57.2 \\
\hline $\mathcal{R}_{5}, \Delta_{5}=-45 \mathrm{dBm}$ & 13.7 & 14.1 & & 8 \\
\hline
\end{tabular}

\begin{tabular}{|c|c|c|c|c|c|c|c|c|c|c|}
\hline \multirow[b]{2}{*}{$\begin{array}{c}\text { EIRP } \\
\text { (in } \mathrm{dBm} \text { ) }\end{array}$} & \multicolumn{4}{|c|}{$\mathcal{R}_{1}$} & \multicolumn{4}{|c|}{$\mathcal{R}_{5}$} & \multicolumn{2}{|c|}{ Improvement } \\
\hline & $\begin{array}{c}\text { Mean } \\
\text { (in } \mathrm{dB})\end{array}$ & $\begin{array}{l}\text { Median } \\
\text { (in } \mathrm{dB} \text { ) }\end{array}$ & $\begin{array}{l}\text { Std. dev. } \\
\text { (in } \mathrm{dB} \text { ) }\end{array}$ & $\begin{array}{c}\% \text { of sph. } \\
\text { (in } \% \text { ) }\end{array}$ & $\begin{array}{c}\text { Mean } \\
\text { (in dB) }\end{array}$ & $\begin{array}{l}\text { Median } \\
\text { (in dB) }\end{array}$ & $\begin{array}{l}\text { Std. dev. } \\
\text { (in } \mathrm{dB} \text { ) }\end{array}$ & $\begin{array}{c}\% \text { of sph. } \\
\text { (in } \% \text { ) }\end{array}$ & $\begin{array}{c}\text { Abs. } \\
\text { (in } \% \text { ) }\end{array}$ & $\begin{array}{l}\text { Rel. } \\
\text { (in \%) }\end{array}$ \\
\hline$>-35$ & 14.7 & 15.6 & 8.3 & 29.7 & 13.9 & 15.4 & 9.2 & 30.8 & 1.1 & 3.7 \\
\hline$>-40$ & 15.1 & 15.7 & 8.9 & 54.7 & 14.2 & 15.4 & 9.9 & 57.2 & 2.5 & 4.6 \\
\hline$>-45$ & 14.1 & 14.3 & 9.1 & 80.1 & 13.7 & 14.1 & 9.6 & 81.8 & 1.7 & 2.1 \\
\hline
\end{tabular}

grip case. Table $\mathrm{V}$ shows that (unlike the hard hand grip case where only $1 \%-2.5 \%$ improvement in spherical coverage was observed with $\mathcal{R}_{5}$ over $\mathcal{R}_{1}$ ) we observe a $2 \%-6.5 \%$ improvement in absolute spherical coverage with $\mathcal{R}_{5}$, which is substantial. The primary reasons for this enhancement are the gains with hand reflections which are captured by $\mathcal{R}_{5}$, but not with $\mathcal{R}_{1}$, illustrating the need for such an enhanced RoI definition.

\section{B. Study 3: $2 \times 1$ Dipole Subarray with a Hard Hand Grip}

We now consider the beamformed performance of the $2 \times 1$ dipole array (in Module 3) with a hard hand grip. For the dipoles, we again consider a codebook of three beams steering energy towards the boresight of the array, $+45^{\circ}$ to the boresight and $-45^{\circ}$ to the boresight, respectively. The change from $\pm 30^{\circ}$ for the $4 \times 1$ subarray to $\mathrm{a} \pm 45^{\circ}$ for the $2 \times 1$ subarray is due to beamwidth differences for differentsized arrays. The beam patterns of these three beams in Freespace appear regular (not illustrated here pictorially due to space constraints) with the beamwidth of each beam being $\approx 40^{\circ}-45^{\circ}$ (which is as expected for a $2 \times 1$ subarray [25]). The beam patterns with the hand phantom and a true hand show significant distortions (again not illustrated pictorially) introduced by the hand and the poor reproducibility of the hand behavior by the hand phantom.

Reflecting these observations, the overlay plot of the beam patterns of the three best beams is plotted in Fig. 8,a), which shows a small ocean of yellow (or high signal strength regions). In comparison, the CDFs of the EIRPs in Fig. 8(b) shows an almost constant gap of $\approx 15 \mathrm{~dB}$ between the Freespace and true hand holding performance. More precisely, Table IV shows that $35 \%-75 \%$ of the spherical coverage is lost at different EIRP levels, or an equivalent
15-20 dB loss at different spherical coverage levels. As with the patches case, $\mathcal{R}_{5}$ does not seem to bring in any additional value over $\mathcal{R}_{1}$ showing that hand reflections are not important with the hard hand grip.

\section{Study 4: $2 \times 1$ Dipole Subarray with a Loose Hand Grip}

In the loose hand grip mode, the $2 \times 1$ dipole subarray shows a similar behavior as the $4 \times 1$ patch subarray with a loose hand grip (Study 2). At different EIRP levels, a $9 \%-20 \%$ spherical coverage is lost relative to the Freespace case corresponding to a $0-11 \mathrm{~dB}$ blockage loss at different spherical coverage levels. The $\mathrm{CDF}$ with the hand phantom is similar to the Freespace scenario showing the poor match of the hand phantom in capturing true blockage performance. In contrast to the hard hand grip case, we see that $\mathcal{R}_{5}$ can result in substantial coverage improvement over $\mathcal{R}_{1}$ (of $6.5 \%-8 \%$ absolute improvement or $8.5 \%-13 \%$ relative improvement). This study shows that hand reflections with loose hand grips need to be carefully captured with RoI such as $\mathcal{R}_{5}$ and a RoI such as $\mathcal{R}_{1}$ is not sufficient in such scenarios.

\section{Study 5: $4 \times 1$ Patch Subarray with an Intermediate Hand Grip}

In the final study, a two-handed grip in the Landscape mode over the $4 \times 1$ patch subarray is considered. This study shows some effects observed with the hard hand grip as well as some effects observed with the loose hand grip studies. In terms of EIRP losses, we see a loss of 9.5-13 dB at different spherical coverage levels, which is equivalent to a spherical coverage loss of $25 \%-50 \%$ at different EIRP levels. This observation is similar to those made in the hard hand grip cases (Studies 1 and 3). On the other hand, 


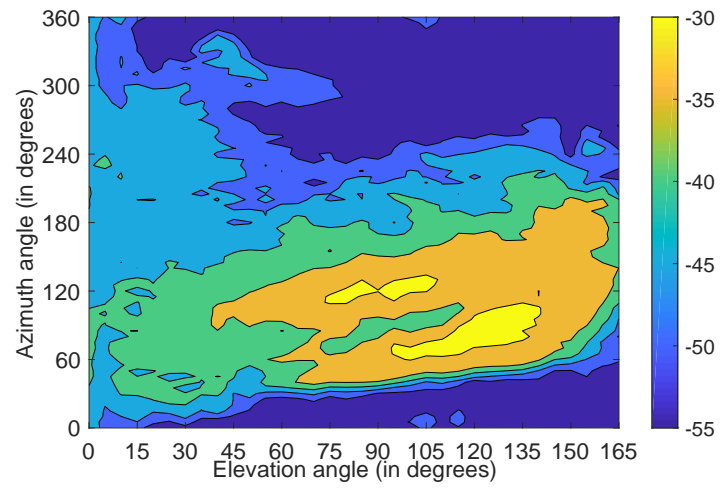

(a)

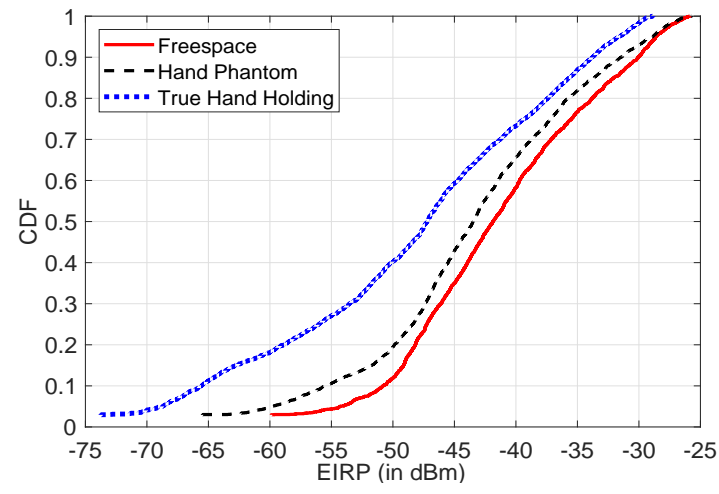

(b)

Fig. 7. (a) Overlay plot of the best of the three beam patterns in Freespace with the $4 \times 1$ patch subarray in loose hand grip mode. (b) CDFs of EIRP in the $4 \times 1$ patch subarray with loose hand grip.

TABLE IV

EIRP-BASED COMPARISONS FOR OTHER STUDIES

\begin{tabular}{|c|c|c|c|c|c|c|c|c|c|c|c|}
\hline \multirow{6}{*}{$\begin{array}{l}\text { N } \\
\text { 齐 } \\
\vec{E}\end{array}$} & & \multicolumn{7}{|c|}{ Spherical Coverage Lost } & \multicolumn{3}{|c|}{ Loss (in $\mathrm{dB}$ ) } \\
\hline & \multirow{2}{*}{$\begin{array}{c}\text { EIRP } \\
\text { (in } \mathrm{dBm} \text { ) }\end{array}$} & \multirow{2}{*}{$\begin{array}{c}\text { Freespace } \\
\text { (in \%) }\end{array}$} & \multicolumn{3}{|c|}{ Hand Phantom (in \%) } & \multicolumn{3}{|c|}{ True Hand (in \%) } & \multirow{2}{*}{$\begin{array}{c}\text { Percentile } \\
90\end{array}$} & \multirow{2}{*}{$\begin{array}{c}\text { Hand Phantom } \\
1.4\end{array}$} & \multirow{2}{*}{$\begin{array}{c}\text { True Hand } \\
3.7\end{array}$} \\
\hline & & & & Abs. & Rel. & & Abs. & Rel. & & & \\
\hline & $>-35$ & 23.3 & 18.1 & 5.1 & 22.0 & 13.1 & 10.2 & 43.9 & 80 & 2.0 & 3.6 \\
\hline & $>-40$ & 41.8 & 34.4 & 7.4 & 17.7 & 26.8 & 15.0 & 36.0 & 50 & 1.6 & 5.4 \\
\hline & $>-45$ & 65.0 & 57.2 & 7.8 & 12.0 & 40.7 & 24.3 & 37.4 & 20 & 1.7 & 10.6 \\
\hline \multirow{4}{*}{$\begin{array}{l}\text { m } \\
\vec{Z} \\
\vec{D}\end{array}$} & & & & & & & & & $\overline{90}$ & 2.6 & $\overline{15.9}$ \\
\hline & $>-40$ & 35.3 & 22.1 & 13.2 & 37.3 & 0.0 & 35.3 & 100.0 & 80 & 2.7 & 16.3 \\
\hline & $>-45$ & 58.9 & 46.4 & 12.5 & 21.3 & 0.7 & 58.2 & 98.8 & 50 & 2.6 & 15.9 \\
\hline & $>-50$ & 84.1 & 69.9 & 14.1 & 16.8 & 7.7 & 76.4 & 90.8 & 20 & 4.2 & 19.7 \\
\hline \multirow{4}{*}{$\begin{array}{l}+ \\
\vec{Z} \\
\vec{E} \\
\tilde{n}\end{array}$} & & & & & & & & & $\overline{90}$ & $\overline{0.4}$ & $\overline{0.4}$ \\
\hline & $>-40$ & 35.3 & 35.3 & 0.0 & 0.0 & 26.2 & 9.1 & 25.7 & 80 & 0.2 & 1.5 \\
\hline & $>-45$ & 58.9 & 61.5 & -2.5 & -4.4 & 47.1 & 11.8 & 20.0 & 50 & -0.4 & 2.6 \\
\hline & $>-50$ & 84.1 & 79.6 & 4.5 & 5.3 & 64.5 & 19.5 & 23.2 & 20 & 1.5 & 10.8 \\
\hline \multirow{4}{*}{ 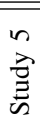 } & & & & & & & & & $\overline{90}$ & $\overline{10.5}$ & 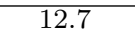 \\
\hline & $>-45$ & 28.7 & 2.7 & 26.1 & 90.7 & 0.04 & 28.7 & 99.9 & 80 & 10.8 & 12.4 \\
\hline & $>-50$ & 50.3 & 11.7 & 38.6 & 76.8 & 7.6 & 42.8 & 85.0 & 50 & 10.7 & 10.9 \\
\hline & $>-55$ & 70.7 & 26.5 & 44.2 & 62.5 & 22.2 & 48.5 & 68.6 & 20 & 9.8 & 9.5 \\
\hline
\end{tabular}

mirroring Studies 2 and 4 (loose hand grip cases), $\mathcal{R}_{5}$ leads to a substantial improvement over $\mathcal{R}_{1}$ of $3.5 \%-10 \%$ absolute spherical coverage increase with hand reflections (corresponding to a relative improvement of 9\%-16\%). Thus, in this study, we see that the hand position/grip leads to a substantial performance decrease over the intended coverage of the subarray, but also a substantial performance increase over other regions of the sphere where the subarray is not intended to cover. Such aspects of blockage need to be carefully considered in understanding the implications of blockage in practical settings.

\section{MOdELS FOR BLOCKAGE AND ITS IMPACT ON PHYSICAL LAYER PERFORMANCE}

We now explore good stochastic model fits for signal strength changes with hand/body blockage and what these models imply for physical layer performance.
Towards this goal, Fig. 9 (a) first plots the CDF of blockage loss defined as the signal strength difference between Freespace and true hand holding scenarios in the five studies with $\mathcal{R}_{5}$ leading to the RoI in these studies. To augment Fig. 9(a), Table VI illustrates the statistics of blockage loss in these five studies along with the parameters that go into the RoI definitions. In addition to plotting the empirical loss data, a simple Gaussian fit with the mean and standard deviation of the data is also plotted for each of the five studies in Fig. 9(a). As mentioned earlier, we note that the mean of blockage loss is substantially less in all the five cases (even with the hard hand grip) relative to prior works that reported loss often in excess of $30 \mathrm{~dB}$. The sources of discrepancies for such wide variations could include beamwidth differences between phased array of antennas in commercial form-factor UEs relative to horn antenna studies that have been reported in prior works, material property differences between UEs and horns, reflections due to hand 
TABLE V

Blockage Performance of $\mathcal{R}_{1}$ And $\mathcal{R}_{5}$ FOR Other Studies

\begin{tabular}{|c|c|c|c|c|c|c|c|c|c|c|c|}
\hline \multirow{5}{*}{$\begin{array}{l}\text { D } \\
\vec{D} \\
\stackrel{\Xi}{n}\end{array}$} & \multirow[b]{2}{*}{$\begin{array}{c}\text { EIRP } \\
\text { (in } \mathrm{dBm} \text { ) }\end{array}$} & \multicolumn{4}{|c|}{$\mathcal{R}_{1}$} & \multicolumn{4}{|c|}{$\mathcal{R}_{5}$} & \multicolumn{2}{|c|}{ Improvement } \\
\hline & & $\begin{array}{c}\text { Mean } \\
\text { (in } \mathrm{dB} \text { ) }\end{array}$ & $\begin{array}{l}\text { Median } \\
\text { (in dB) }\end{array}$ & $\begin{array}{l}\text { Std. dev. } \\
\text { (in dB) }\end{array}$ & $\begin{array}{c}\% \text { of sph. } \\
\text { (in } \% \text { ) }\end{array}$ & $\begin{array}{c}\text { Mean } \\
\text { (in dB) }\end{array}$ & $\begin{array}{l}\text { Median } \\
\text { (in } \mathrm{dB} \text { ) }\end{array}$ & $\begin{array}{l}\text { Std. dev. } \\
\text { (in } \mathrm{dB} \text { ) }\end{array}$ & $\begin{array}{c}\text { \% of sph. } \\
\text { (in } \% \text { ) }\end{array}$ & $\begin{array}{c}\text { Abs. } \\
\text { (in \%) }\end{array}$ & $\begin{array}{l}\text { Rel. } \\
\text { (in \%) }\end{array}$ \\
\hline & $>-35$ & 5.1 & 4.1 & 4.6 & 29.7 & 4.2 & 3.8 & 5.9 & 31.7 & 2.0 & 6.7 \\
\hline & $>-40$ & 7.5 & 5.3 & 7.5 & 54.7 & 6.4 & 4.7 & 8.6 & 58.4 & 3.7 & 6.8 \\
\hline & $>-45$ & 7.3 & 4.9 & 8.1 & 80.1 & 5.8 & 4.3 & 9.4 & 86.5 & 6.4 & 8.0 \\
\hline \multirow{3}{*}{ 㞷 } & $>-40$ & 19.3 & 18.4 & 8.1 & 51.3 & 19.3 & 18.4 & 8.1 & 51.3 & 0 & 0 \\
\hline & $>-45$ & 18.6 & 18.2 & 8.0 & 72.7 & 18.6 & 18.2 & 8.0 & 72.7 & 0 & 0 \\
\hline & $>-50$ & 17.7 & 17.6 & 8.0 & 87.1 & 17.2 & 17.3 & 9.0 & 88.6 & 1.5 & 1.7 \\
\hline \multirow{3}{*}{ 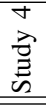 } & $>-40$ & 3.7 & 0.4 & 7.5 & 51.3 & 1.5 & 0.04 & 10.4 & 57.9 & 6.6 & 12.9 \\
\hline & $>-45$ & 4.8 & 0.8 & 8.3 & 72.7 & 2.9 & 0.4 & 10.5 & 80.5 & 7.8 & 10.7 \\
\hline & $>-50$ & 5.1 & 1.3 & 8.4 & 87.2 & 3.4 & 0.7 & 10.4 & 94.6 & 7.4 & 8.5 \\
\hline \multirow{3}{*}{  } & $>-45$ & 18.3 & 18.4 & 5.6 & 39.5 & 15.9 & 17.9 & 9.5 & 43.0 & 3.5 & 8.9 \\
\hline & $>-50$ & 14.9 & 16.6 & 7.8 & 63.9 & 11.3 & 15.5 & 11.8 & 73.9 & 10.0 & 15.7 \\
\hline & $>-55$ & 13.1 & 14.6 & 8.4 & 81.8 & 10.5 & 13.6 & 11.3 & 90.8 & 9.0 & 11.0 \\
\hline
\end{tabular}

TABLE VI

Statistics of Blockage Loss in the Five Studies

\begin{tabular}{|c||c|c|c|c|c||}
\hline Study & $\Delta_{5}$ (in dBm) & Mean (in dB) & Median (in dB) & Std. deviation (in dB) & Percentage of sphere \\
\hline 1 & -35 & 13.9 & 15.4 & 9.2 & $30.8 \%$ \\
\hline 2 & -35 & 4.2 & 3.8 & 5.9 & $31.7 \%$ \\
\hline 3 & -40 & 19.3 & 18.4 & 8.1 & $51.3 \%$ \\
\hline 4 & -40 & 1.5 & 0.04 & 10.4 & $57.9 \%$ \\
\hline 5 & -45 & 15.9 & 17.9 & 9.5 & $43.0 \%$ \\
\hline \hline
\end{tabular}

that is often unaccounted for in prior works, etc. Fig. 9 (a) also shows that while simple Gaussian models are good for hard/intermediate hand grips with substantial losses, looser hand grips with a steeper loss curve and wider tails need more sophisticated multi-parameter models such as Gamma distribution, Weibull distribution, etc [22]. Empirical fits of such distributions to data is the subject of ongoing work and will be reported elsewhere.

We now study the implications of blockage loss from true hand holding on beamforming performance relative to models from prior works. For this, Fig. 9(b) plots the EIRP distribution seen over the Freespace scenario and the true hand holding in Studies 1 and 2 (hard hand grip vs. loose hand grip mode) with the $\mathcal{R}_{5}$ RoI and by setting $\Delta_{5}=-35 \mathrm{dBm}$. Also, plotted are the EIRP deteriorations due to the 3GPP model [13] and the model from [22] for this RoI. Clearly, we note that the 3GPP model widely overestimates the blockage loss even in the hard hand grip case. On the other hand, the model from [22] has a comparable performance to the hard hand grip case, whereas it overestimates the blockage loss in the loose hand grip case. Even within the hard hand grip case, the model from [22] does not capture the hand reflections and thus there is a cross-over between the CDFs observed here and the model from [22] (better true performance at peak coverage points and weaker true performance at lower tails). Such a crossover can lead to a poor estimation of EIRP (and thus physical layer performance) in a practical context, which requires careful study such as the one in this work.

\section{CONCLUding Remarks}

The focus of this paper has been on understanding hand/body blockage with commercial quality phased arrays in a user equipment operating at $28 \mathrm{GHz}$. For this, a number of controlled studies were performed and the impact of blockage was estimated with hard, intermediate and loose hand grips. Our studies showed that blockage produces a complex effect on the received signal strength depending on the direction of interest. In the main scenario (also addressed in prior works), blockage leads to signal strength deterioration. But unlike estimates from prior works which are excessive, we show that this deterioration is moderate $(<5 \mathrm{~dB}$ for loose hand grip) to reasonable $(<15 \mathrm{~dB}$ for intermediate to hard hand grips). Additionally, with looser hand grips and based on the hand holding, signals can be reflected by the fingers, palm and different parts of the hand to improve signal strengths in hitherto weak signal directions (as seen from a Freespace perspective). Such a complicated behavior has not been explored or illustrated in prior works and this work documents such hand reflection gains.

In terms of future work, this paper exposes the need for further careful studies in understanding how blockage can affect millimeter wave devices. Given that blockage is expected to have a serious effect on the link budget 


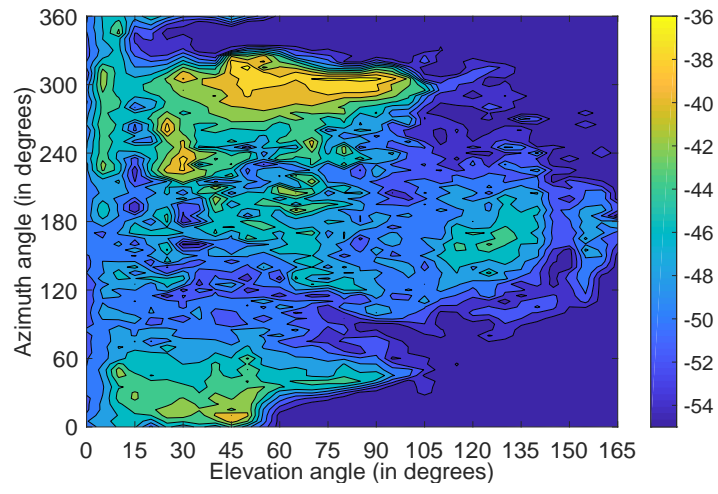

(a)

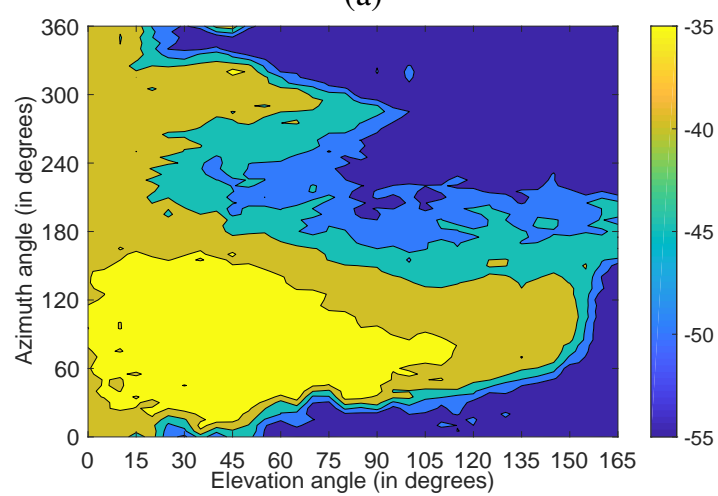

(b)



(c)

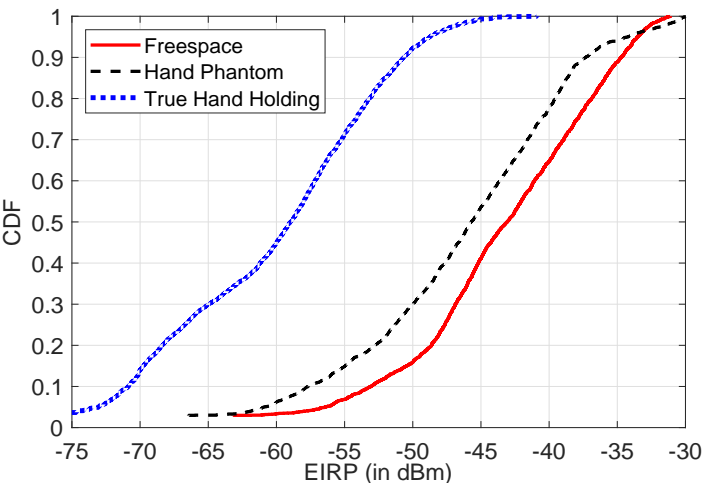

(d)

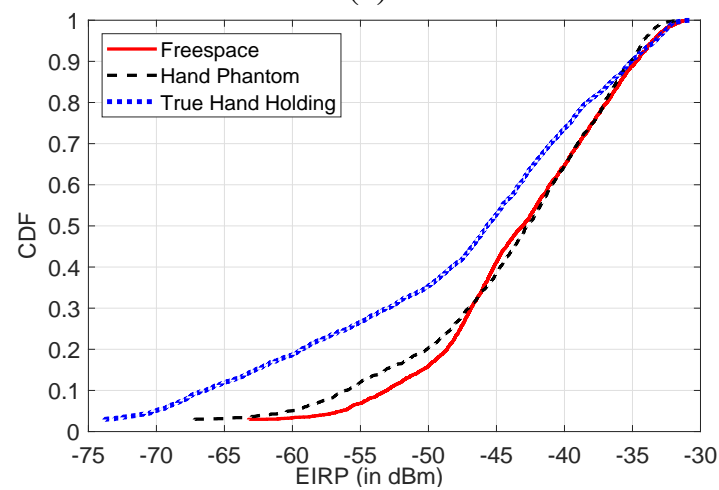

(e)

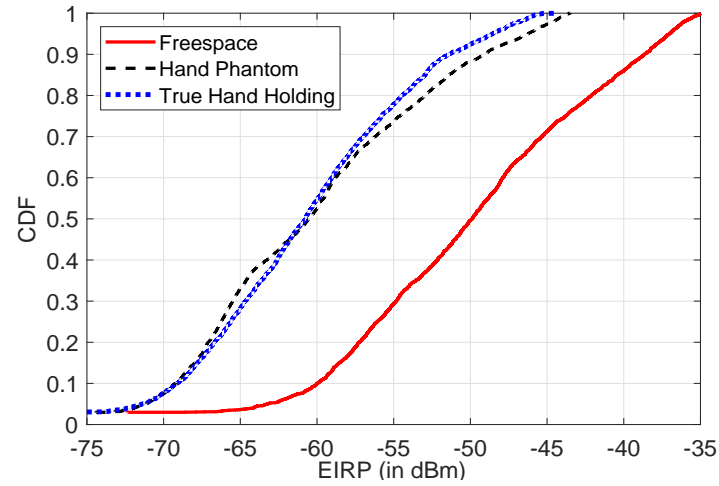

(f)

Fig. 8. (a)-(c) Overlay plot of the best of the three beam patterns in Freespace for Studies 3-5. (d)-(f) CDFs of EIRP with different hand modes in Studies 3-5.

of commercial/cellular systems, a number of system level design questions become pertinent. It is important to understand how different user hand grips/holdings can affect signal strength behavior (perhaps generate some parametric models to capture these effects), how blockage plays into network densification questions, and the role of mitigation mechanisms such as multi-panel, multi-beam and cooperative schemes. Also, important to understand is the costpower-performance tradeoffs in the use of multiple antenna modules at millimeter wave frequencies [26]. Yet another broad question of interest is the tuning of hand phantoms to match true hand holding results.

\section{REFERENCES}

[1] F. Rusek, D. Persson, B. K. Lau, E. G. Larsson, T. L. Marzetta, O. Edfors, and F. Tufvesson, "Scaling up MIMO: Opportunities and challenges with very large arrays," IEEE Sig. Proc. Magaz., vol. 30, no. 1, pp. 40-60, Jan. 2013.

[2] S. Hur, T. Kim, D. J. Love, J. V. Krogmeier, T. A. Thomas, and A. Ghosh, "Millimeter wave beamforming for wireless backhaul and access in small cell networks," IEEE Trans. Commun., vol. 61, no. 10, pp. 4391-4403, Oct. 2014.

[3] S. Sun, T. S. Rappaport, R. W. Heath, Jr., A. Nix, and S. Rangan, "MIMO for millimeter wave wireless communications: Beamforming, spatial multiplexing, or both?," IEEE Commun. Magaz., vol. 52, no. 12, pp. 110-121, Dec. 2014.

[4] J. Brady, N. Behdad, and A. M. Sayeed, "Beamspace MIMO for millimeter-wave communications: System architecture, modeling, 




(a)

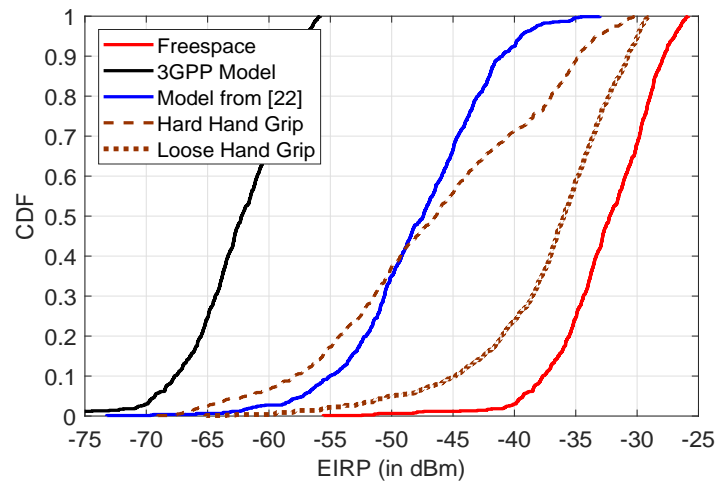

(b)

Fig. 9. (a) CDF of blockage loss with $\mathcal{R}_{5}$ in all the five studies. (b) EIRP comparison over $\mathcal{R}_{5}$ RoI with Freespace and different blockage models.

analysis and measurements," IEEE Trans. Ant. Propagat., vol. 61, no. 7, pp. 3814-3827, July 2013.

[5] O. El Ayach, S. Rajagopal, S. Abu-Surra, Z. Pi, and R. W. Heath, Jr., "Spatially sparse precoding in millimeter wave MIMO systems," IEEE Trans. Wireless Commun., vol. 13, no. 3, pp. 1499-1513, Mar. 2014.

[6] V. Raghavan, J. Cezanne, S. Subramanian, A. Sampath, and O. H. Koymen, "Beamforming tradeoffs for initial UE discovery in millimeter-wave MIMO systems," IEEE Journ. Sel. Topics in Sig. Proc., vol. 10, no. 3, pp. 543-559, Apr. 2016.

[7] V. Raghavan, S. Subramanian, J. Cezanne, A. Sampath, O. H. Koymen, and J. Li, "Single-user vs. multi-user precoding for millimeter wave MIMO systems," IEEE Journ. Sel. Areas in Commun., vol. 35, no. 6, pp. 1387-1401, June 2017.

[8] W. Roh, J.-Y. Seol, J. Park, B. Lee, J. Lee, Y. Kim, J. Cho, K. Cheun, and F. Aryanfar, "Millimeter-wave beamforming as an enabling technology for $5 \mathrm{G}$ cellular communications: Theoretical feasibility and prototype results," IEEE Commun. Magaz., vol. 52, no. 2, pp. 106-113, Feb. 2014.

[9] S. Rangan, T. S. Rappaport, and E. Erkip, "Millimeter wave cellular networks: Potentials and challenges," Proc. IEEE, vol. 102, no. 3, pp. 366-385, Mar. 2014.

[10] A. Ghosh, T. A. Thomas, M. C. Cudak, R. Ratasuk, P. Moorut, F. W. Vook, T. S. Rappaport, G. R. MacCartney, Jr., S. Sun, and S. Nie, "Millimeter-wave enhanced local area systems: A high datarate approach for future wireless networks," IEEE Journ. Sel. Areas in Commun., vol. 32, no. 6, pp. 1152-1163, June 2014.

[11] V. Raghavan, A. Partyka, S. Subramanian, A. Sampath, O. H. Koymen, K. Ravid, J. Cezanne, K. K. Mukkavilli, and J. Li, "Millimeter wave MIMO prototype: Measurements and experimental results," IEEE Commun. Magaz., vol. 56, no. 1, pp. 202-209, Jan. 2018.

[12] A. Maltsev et al., "Channel models for $60 \mathrm{GHz}$ WLAN systems, doc: IEEE 802.11-09/0334r8," May 2010, Available: [Online]. https://mentor.ieee.org/802.11/documents.

[13] 3GPP TR 38.901 V14.3.0 (2017-12), "Technical Specification Group Radio Access Network; Study on Channel Model for Frequencies from 0.5 to $100 \mathrm{GHz}$ (Rel. 14)," Dec. 2017.

[14] METIS 2020, "METIS channel model, Deliverable D1.4v3," July 2015, Available: [Online] https://www.metis2020.com/wp-content/uploads/ deliverables/METIS_D1.4_v3.pdf.

[15] M. Peter, M. Wisotzki, M. Raceala-Motoc, W. Keusgen, R. Felbecker, M. Jacob, S. Priebe, and T. Kuerner, "Analyzing human body shadowing at $60 \mathrm{GHz}$ : Systematic wideband MIMO measurements and modeling approaches," Proc. European Conf. Ant. Propagat., Prague, Czech Republic, pp. 468-472, Mar. 2012.

[16] G. R. MacCartney, Jr., S. Deng, S. Sun, and T. S. Rappaport, "Millimeter-wave human blockage at $73 \mathrm{GHz}$ with a simple double knife-edge diffraction model and extension for directional antennas,"
Proc. IEEE Veh. Tech. Conf. (Fall), Montreal, Canada, pp. 1-6, Sept. 2016.

[17] G. R. MacCartney, Jr. and T. S. Rappaport, "A flexible millimeterwave channel sounder with absolute timing," IEEE Journ. Sel. Areas in Commun., vol. 35, no. 6, pp. 1402-1418, June 2017.

[18] Aalto University, AT\&T, BUPT, CMCC, Ericsson, Huawei, Intel, KT Corporation, Nokia, NTT DOCOMO, NYU, Qualcomm, Samsung, U. Bristol, and USC, "White paper on "5G channel model for bands up to $100 \mathrm{GHz},, \quad v 2.3$, Oct. 2016, Available: [Online]. http://www . 5gworkshops . com/5GCM. html.

[19] T. S. Rappaport, G. R. MacCartney, Jr., S. Sun, H. Yan, and S. Deng, "Small-scale, local area, and transitional millimeter wave propagation for 5G communications," IEEE Journ. Ant. Propagat., vol. 65, no. 12, pp. 6474-6490, Dec. 2017.

[20] G. R. MacCartney, Jr., T. S. Rappaport, and S. Rangan, "Rapid fading due to human blockage in pedestrian crowds at $5 \mathrm{G}$ millimeter-wave frequencies," Proc. IEEE Global Telecommun. Conf., Singapore, pp. 1-7, Dec. 2017.

[21] K. Zhao, J. Helander, Z. Ying, D. Sjöberg, M. Gustafsson, and S. He, "mmWave phased array in mobile terminal for 5G mobile system with consideration of hand effect," Proc. IEEE Veh. Tech. Conf. (Spring), Glasgow, Scotland, pp. 1-4, May 2015.

[22] V. Raghavan, L. Akhoondzadeh-Asl, V. Podshivalov, J. Hulten, M. A. Tassoudji, O. H. Koymen, A. Sampath, and J. Li, "Statistical blockage modeling and robustness of beamforming in millimeter wave systems," IEEE Trans. Microwave Theory and Tech., vol. 67, no. 7, pp. 3010-3024, July 2019.

[23] 3GPP Specification Series, "Technical Specifications, 38 Series," 2017, Available: [Online]. https://www.3gpp.org/DynaReport /38-series.htm.

[24] V. Raghavan, S. Subramanian, J. Cezanne, and A. Sampath, "Directional beamforming for millimeter-wave MIMO systems," Proc. IEEE Global Telecommun. Conf., San Diego, CA, pp. 1-7, Dec. 2015.

[25] C. A. Balanis, Antenna Theory: Analysis and Design, WileyInterscience, 3rd edition, 2005.

[26] V. Raghavan, M-L. (Clara) Chi, M. A. Tassoudji, O. H. Koymen, and J. Li, "Antenna placement and performance tradeoffs with hand blockage in millimeter wave systems," IEEE Trans. Commun., vol. 67, no. 4, pp. 3082-3096, Apr. 2019. 